\title{
On the Detection of Possible Landslides in Post-Event Satellite Images: A Probability Map Approach
}

\author{
Mohammad Faizal Ahmad Fauzi, Agustinus Deddy Arief Wibowo, Sin Liang Lim, and Wooi Nee Tan
}

\begin{abstract}
Landslides are a significant hazard to property and livelihoods, causing millions of dollars worth of damage annually throughout the world, but especially in tropical regions such as Malaysia. Automated or semi-automated detection of landslides from aerial or satellite imagery and generating landslide susceptibility or hazard map are two of the main research topics in landslide research. In this paper, we propose a probability map approach in detecting possible landslide regions from satellite or aerial images. The detected landslides, tabulated as landslide inventory map, will be useful as the ground truth for evaluating landslide susceptibility map, or even used as one of the causative factors for the susceptibility map itself. The proposed probability map is computed using only colour information, but demonstrated very promising performance in locating potential landslide regions; thus provides a strong platform to locate actual landslides by incorporating texture and shape features in the future.
\end{abstract}

Index Terms-Landslide detection, landslide inventory, landslide probability map.

\section{INTRODUCTION}

Landslide is one of the harzardous phenomena frequently occured in many parts of the world. In Malaysia, landslides are normal occurrence and happen quite freqently due to annual monsoon seasons. Landslide susceptibility mapping is a study for early detection of possible landslide occurences. Dataset containing past landslide occurence points, terrain parameters, geological attributes collected through geographical information system [1], [2], weather data, satellite or airborne laser altimeter imaginery [3]-[8] are among the data used in creating susceptibility maps. In recent years, several research works have also been carried out to create models for landslide detection and prediction for a few locations in Malaysia, such as Cameron Highlands [9] and Penang [10], [11] with varying goals as well as degree of success. It is well-noted that creating an automated landslide prediction model that is able to dynamically perform within uncertain parameters, such as rainfall or weather forecast is a huge challenge.

Automated detection of landslide to create a landslide inventory map is very useful in producing a reliable landslide

Manuscript received November 12, 2014; revised February 26, 2015. This work was supported in part by the Malaysian Ministry of Higher Education under Grant ERGS/1/2012/STWN01/MMU/02/2.

Mohammad Faizal Ahmad Fauzi, Sin Liang Lim, and Wooi Nee Tan are with the Faculty of Engineering, Multimedia University, Cyberjaya, Selangor, Malaysia (e-mail: faizal1@mmu.edu.my, lim.sin.liang@mmu.edu.my,wntan@mmu.edu.my).

Agustinus Deddy Arief Wibowo was with the Faculty of Engineering, Multimedia University, Cyberjaya, Selangor, Malaysia (e-mail: deddy_0708@yahoo.com). susceptibility map. The inventory map can either be used as the ground truth to evaluate the performance of a susceptibility map, or as one of the causative factors (by calculating distance to past landslides) themselves alongside vegetation index, lithology, slope structure, slope aspect, distance to rivers, distance to roads, soil type and rainfall data.

Landslide inventory has traditionally been completed using aerial or satellite imagery and manual digitizing. However this is a very time consuming task and capturing these data over broad areal extents is often not feasible. Alternatively automated or semi-automated detection methods using aerial or satellite images can provide information in a more time-efficient manner. The detection method can be based on either single set of images taken at a particular time, or multiple sets of images taken before and after the occurrence of landslides. Most landslide detection algorithms often opt for the former where the pre- and post-event data are required [12]-[14]. Unfortunately it is often difficult to obtain such data, not to mention the additional work required to register the pre- and post-event images. Using only post-event satellite image, while very challenging, would be a very valuable tool in landslide research, especially in the absence of digital elevation model (DEM) data.

In this paper, we present our preliminary results in detecting potential landslide regions from single post-even satellite images by means of a probability map generated based on the likelihood of a pixel belonging to a landslide. The probability map is computed based on the distance of the pixels to either the orange-ish color of a landslide, or a green-ish color of a terrain, on a shifted and scaled HSV model. The proposed detection is thus based only on color information but nevertheless can produce very promising detection, and provides a strong basis for our future work in incorporating texture and shape features in detecting actual landslides.

This paper is organized as follows. Section II describes the proposed detection technique, where the computation of the probability map via a shifted and scaled HSV color model is explained. Section III presents the experimental results and discussion quantitatively as well as qualitatively. Finally Section IV concludes the paper by summarizing important points and avenues for future work.

\section{Proposed Detection Method}

Our proposed method consists of several stages as shown in Fig. 1. The first step is the probability map computation in a shifted and scaled HSV (Hue-Saturation-Value) colour 
space. The probability map calculates the likelihood of each pixel belonging to either landslide or terrain regions, thus simple thresholding can be used to obtain the potential landslide regions. Post-processing is then required to filter small isolated regions from the segmented image. We will demonstrate that the proposed algorithm performs well with a wide variety of images, and unlike the more common segmentation approach such as clustering (k-means, fuzzy c-means, mean shift etc.), it does not suffer from illumination or lighting problem, as well as able to detect very small regions. It also does not require the number of regions to be provided a priori.

\section{A. Probability Map Computation}

We have previously used a similar probability map approach in segmenting chronic wounds [15] with high success rate. Here, we modify the approach to fit into landslide detection framework.

Landslide generally corresponds to orange or brown colour as opposed to the green nature of the surrounding terrain. We calculate the probability of each pixel in the image belonging to these two colours, resulting in a two-dimensional (2D) probability map. Given a satellite image, our method computes the probability of each pixel in the image belonging to one of these colour categories. The probability is computed based on the distance of the image pixels to the orange and green colours in a shifted and scaled HSV colour space. Potential landslides would have higher probabilities for orange class, whereas terrain regions would have higher probabilities for the green class. Any other objects (roads, rivers, buildings etc.) may project colours in between these two extremities; hence their probabilities of belonging to either of these two classes will be low. For a particular pixel x, the probability $\mathrm{p}$ of the pixel belonging to orange and green class is computed through the following equation:

$$
\begin{gathered}
p_{\text {orange }}(x)=\frac{1}{\left(\frac{d \text { (orange }, x)}{d(\text { green }, x)}\right)^{2}+\left(\frac{d(\text { orange }, x)}{d(\text { or ange }, x)}\right)^{2}} \\
p_{\text {green }}(x)=\frac{1}{\left(\frac{d \text { green }, x)}{d(\text { green }, x)}\right)^{2}+\left(\frac{d \text { green }, x)}{d(\text { orange }, x)}\right)^{2}}
\end{gathered}
$$

where $d$ is the distance between the pixel and the respective reference colours. The sum of the $2 \mathrm{D}$ probability map at any one pixel should equal to 1 . The probability definition used here is similar to that of the fuzzy c-means clustering method but without the fuzzifier parameter.

In the original HSV colour model, the orange (around $30^{\circ}$ ) and green (around $120^{\circ}$ ) hues are rather close to each other on the left side of the gamut, with red hues occupy the two extreme ends. Depending on the illumination, landslides may appear as red, hence having red hues to split into the two extremes may lead to inaccuracies in distance and probability computation. We propose to shift the Hue by $30^{\circ}$ to the right so that all of the red hues will be concentrated on one end, closer to the orange reference point. The scale now has magenta splitting into the two ends, but since satellite images rarely exhibit magenta colours, the computation inaccuracies problem will be minimized or avoided altogether.

Fig. 2 shows the original and shifted versions of the Hue. It can be seen that all the red colours are now closer to the orange compared to the green, while other colours such as blue are further away from the orange so that their probabilities of belonging to potential landslide regions is low. The Hue channel is usually normalized into the range of 0 and 1 . To prevent the distance calculation being dominated by Saturation and Value, we scale the Hue so that the distance between the orange and green reference points is 1 . Since orange and green hues are about $90^{\circ}$ apart, this is equivalent to scaling the Hue by 4 . The shifted and scaled HSV model thus provides a better platform in computing the probability map.

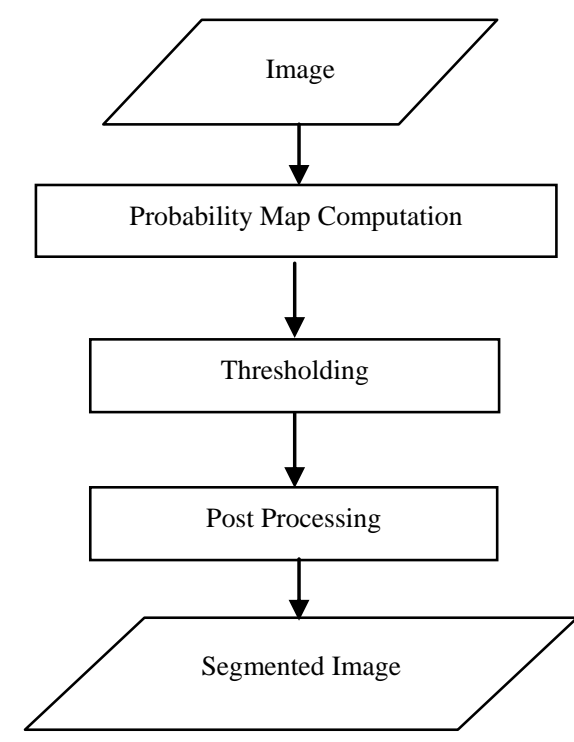

Fig. 1. Flowchart of the proposed method.
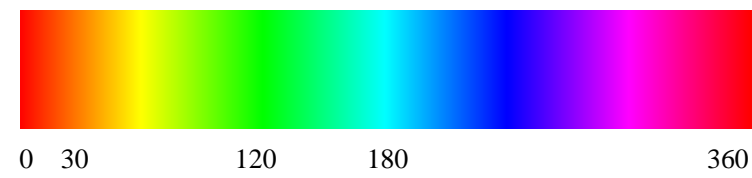

(a)

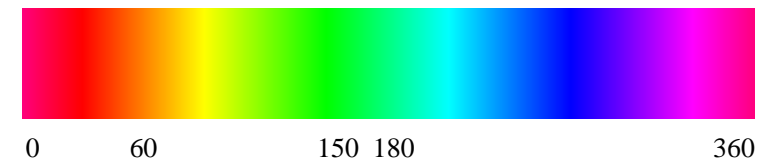

(b)

Fig. 2. (a) Original Hue, (b) Shifted and scaled Hue.

The distance can then be calculated using a standard Euclidean equation, and the probability map can be obtained accordingly by utilising Equations 1 and 2. Table I shows the reference values for the Hue, Saturation and Value used in our work. Fig. 3 shows an example of satellite images with their probability map (only the probability of orange class is shown). Brighter regions indicate higher probability of the regions belonging to a potential landslide region.

\section{B. Thresholding}

The probability map was designed in such a way that pixels in a potential landslide region will be assigned very high probability of belonging to the orange class. Therefore choosing a high enough threshold value would lead to accurate detection of potential landslide regions. From experiment, threshold value of 0.9 is found to be suitable, meaning that only pixels with higher than $90 \%$ probability of belonging to the orange class will be considered. 


\begin{tabular}{lll}
\multicolumn{2}{l}{ TABLE I: REFERENCE VALUES FOR LANDSLIDE AND TERRAIN } \\
\hline & Landslide & Terrain \\
\hline Hue (Original) & $30^{\circ}$ (normalized to & $120^{\circ}$ (normalized to \\
& 0.0833 ) & 0.333 ) \\
Hue (Scaled & $60^{\circ}$ (normalized to & $150^{\circ}$ (normalized to \\
and shifted) & $0.667)$ & $1.667)$ \\
Saturation & 0.25 & 0.25 \\
Value & 1 & 0.5 \\
& & \\
& & \\
& & \\
& & \\
\end{tabular}

\section{Post-Processing}

The detected regions may consist of isolated pixels and small spurious regions, or in the case of satellite image with illumination problem, false regions. Post processing is carried out to filter out these regions by removing all thresholded regions smaller than a particular pixel size, thus ensure that only genuine regions with similar colour characteristics to a landslide are detected. On top of this morphological closing is also used to smooth the boundary as well as closing small gaps within the detected regions.

\section{EXPERIMENTAL RESULTS AND DISCUSSION}

The proposed detection algorithm is applied to several satellite images with one or more potential landslide regions. The selected satellite images also provide different challenges to the algorithm such as images with very small potential landslide regions, as well as images with illumination problem. Overall, 17 images were experimented and the average sensitivity (Equation 3) and specificity (Equation 4) are both above $80 \%$.

$$
\begin{aligned}
& \text { Sensitivity }=\frac{\text { True Positive }}{\text { True Positive }+ \text { False Negative }} \\
& \text { Specificity }=\frac{\text { True Positive }}{\text { True Positive }+ \text { False Positive }}
\end{aligned}
$$

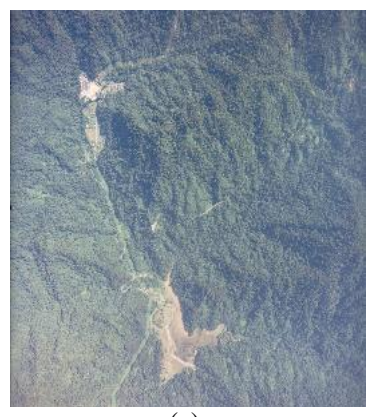

(a)

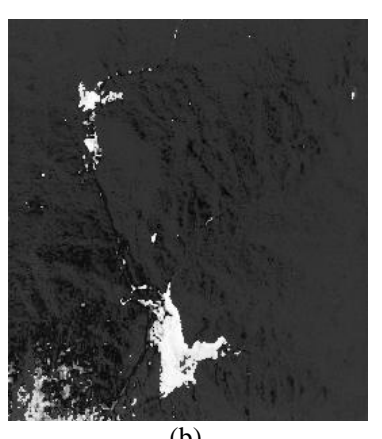

(b)

Fig. 3. (a) Satellite image, (b) Probability map.
Fig. 4 shows a couple of examples of the detection results. The left-hand images are the satellite images, the middle images are the detected potential landslide regions, and the right-hand images are the remaining part of the images not detected as landslides, shown so that readers can judge if there are any potential landslide regions gone undetected. As can be seen, the proposed method manages to detect the orange regions quite accurately. In fact, the second example is the image of the same location but from a different angle, thus demonstrating that the detection method can produce a stable detection regardless of the acquisition angle.

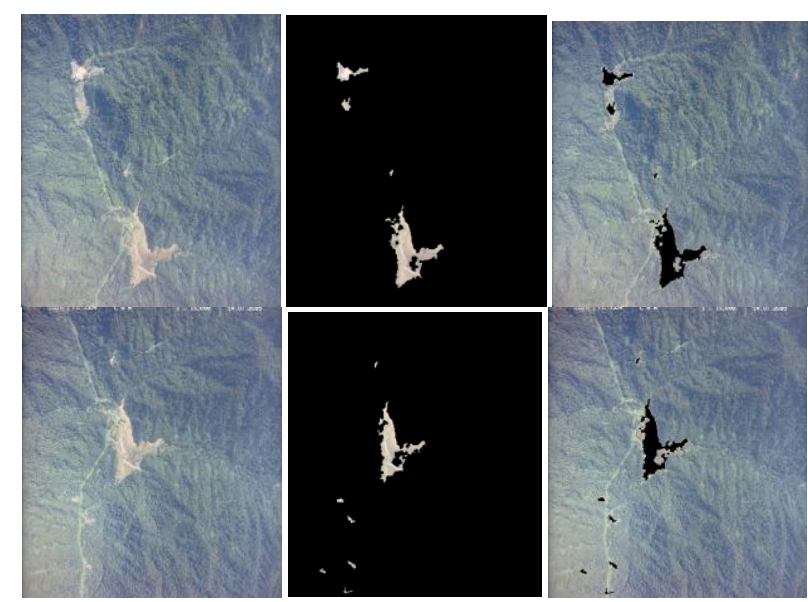

Fig. 4. Example of potential landslide detection results.

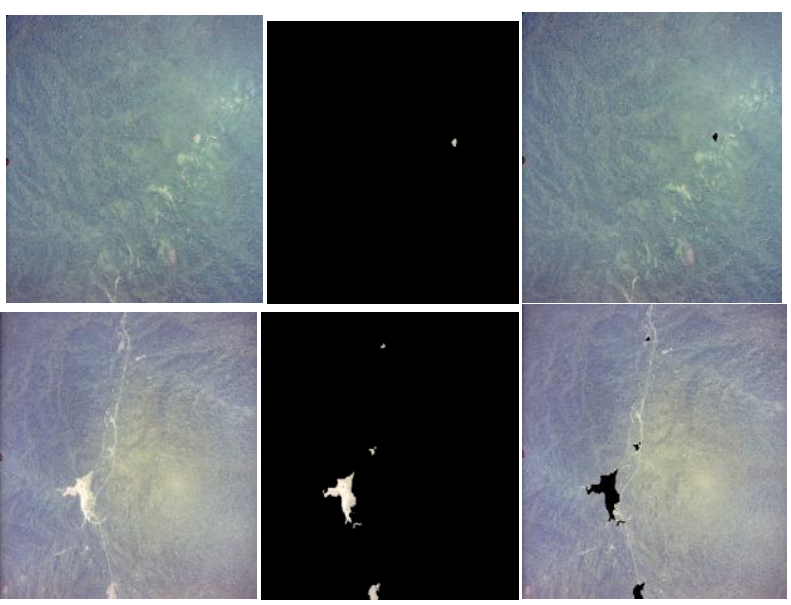

Fig. 5. Example of very small landslide candidates (top) and illumination problem (bottom).

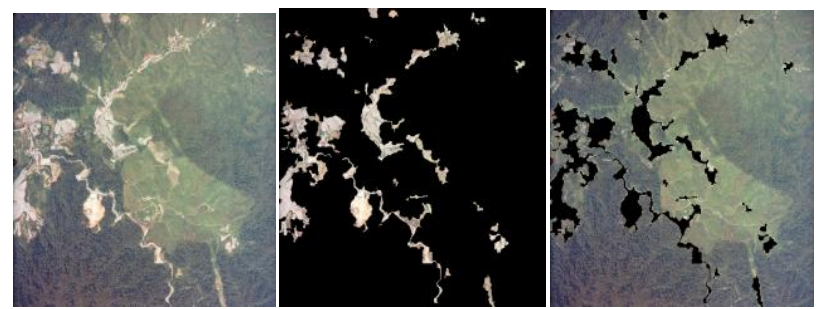

Fig. 6. Example of result on images with complex human activities.

Fig. 5 shows a couple more examples, one of which is an image with very small landslide candidate region, while the other is an image with some illumination problem, producing terrain regions with bright, yellowish region, almost similar to a landslide colour characteristics. For both cases, the proposed method is able to correctly detect the potential 
regions, with no false positives from the bad illumination, and no false negative from the very small candidate region. Figure 6 on the other hand shows how the proposed detection performs when presented with image containing human activities such as deforestation, building or construction sites. The method is able to still differentiate the terrain regions from these regions of interest. While the regions with human activities are also detected together with the candidate landslide regions, we can further distinguish the two later by utilising texture and shape features.

\section{CONCLUSION}

We have proposed a novel method in detecting possible past landslide regions from single post-event satellite images. The method is based on computing the probability map of image pixels belonging to a landslide candidate region, followed by thresholding and post-processing. The probability map is generated from a shifted and scaled HSV colour model where the probability is inversely related to the distance of the pixels to the orange and green reference colours. Experiments on several satellite images posing variety of challenges shows that the proposed method is very reliable in detecting potential landslide regions. We are currently extending the algorithm by incorporating texture and shape features to identify actual landslide regions.

\section{REFERENCES}

[1] A. Carrara, M. Cardinali, R. Detti, F. Guzzetti, V. Pasqui, and P. Reichebach, "GIS techniques and statistical models in evaluating landslide hazard," Earth Surface Processes and Landforms, vol. 16, issue 5, pp. 427-445, 1991.

[2] X. Yao and F. C. Dai, "Support vector machine modeling of landslide susceptibility using a GIS: A case study," in Proc. the 10th IAEG International Congress, United Kingdom, 2006.

[3] A. Sheth, K. Tejaswi, P. Metha, C. Parekh, R. Bansal, S. Merchant, T. N. Singh, U. B. Desai, C. A. Thekkath, and K. Toyama, "Sen slide: A Sensor network based landslide prediction system," in Proc. the 3rd International Conference on Embedded Networked Sensor Systems, 2005, pp. 280-281.

[4] A. Sheth, C. A. Thekkath, P. Mehta, K. Tejaswi, C. Parekh, T. N. Singh, and U. B. Desai, "Senslide: A distributed landslide prediction system," in ACM SIGOPS Operating Systems Review - Systems work at Microsoft Research Archive, vol. 41, issue 2, 2007

[5] J. McKean and J. Roering, "Objective landslide and surface morphology mapping using high-resolution airborne laser altimetry,' Geomorphology, Elservier, vol. 57, issue 3-4, pp 331-351, 2004.

[6] M. V. Ramesh, "Real-time wireless sensor network for landslide detection," in Proc. the 2009 International Conference on Sensor Technologies and Applications, 2009, pp. 405-409.

[7] S. Filin, "Surface classification from airborne laser scanning data," Computers and Geosciences, Elservier, vol. 30, issue 9-10, pp. 1033-1041, 2004.

[8] K. E. Joyce, G. D. Dellow, and P. J. Glassey, "Using remote sensing and spatial analysis to understand landslide distribution and dynamics in New Zealand," in Proc. the IEEE International Geoscience and Remote Sensing Symposium, 2009, vol. III, pp. 224-227.

[9] B. Pradhan, E. A. Sezer, C. Gokgeoglu, and M. F. Buchroithner, "Landslide susceptibility mapping by neuro-fuzzy approach in a landslide-prone area (Cameron Highlands, Malaysia)," IEEE Transactions on Geoscience and Remote Sensing, vol. 48, no. 12, pp 4164-4177, 2010.

[10] B. Pradhan and S. Lee, "Delineation of landslide hazard areas on Penang island, Malaysia, by using frequency ratio, logistic regression, and artificial neural network models," Environment Earth Science, Springer Verlag, vol. 60, no. 5, pp. 1037-1054, 2010.

[11] K. W. D. Lim, L. T. Tay, and H. Lateh, "Landslide hazard mapping of penang island using probabilistic methods and logistic regression," in Proc. the 2011 IEEE International Conference on Imaging Systems and Techniques, 2011, pp. 273-278.

[12] P. Lu, A. Stumpf, N. Kerle, and N. Casagli, "Object-oriented change detection for landslide rapid mapping," IEEE Geoscience And Remote Sensing Letters, vol. 8, issue 4, pp. 701-705, 2011.

[13] F. Tsai, J. H. Hwang, L. C. Chen, and T. H. Lin, "Post-disaster assessment of landslides in southern Taiwan after 2009 Typhoon Morakot using remote sensing and spatial analysis," Natural Hazards and Earth System Sciences, vol. 10, pp. 2179-2190, 2010.

[14] P. L. Rosin, J. Herv'as, and J. Berrado, "Remote sensing image thresholding for landslide motion detection," in Proc. the 1st International Workshop on Pattern Recognition Techniques in Remote Sensing, 2000, pp. 10-17.

[15] M. F. A. Fauzi, I. Khansa, K. Catignani, G. Gordillo, C. K. Sen, and M. N. Gurcan, "Segmentation and automated measurement of chronic wound images: probability map approach," in Proc. the SPIE: Medical Imaging, 2014.

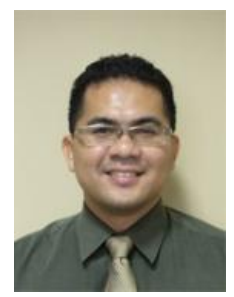

Mohammad Faizal Ahmad Fauzi received the B.Eng. degree in electrical and electronic engineering from Imperial College, London, UK in 1999, and the $\mathrm{Ph} . \mathrm{D}$. degree in electronics and computer science from University of Southampton, Southampton, UK in 2004. He is currently an associate professor at the Faculty of Engineering, Multimedia University. His main research interests are in the area of signal and image processing, pattern recognition, computer vision and medical imaging.

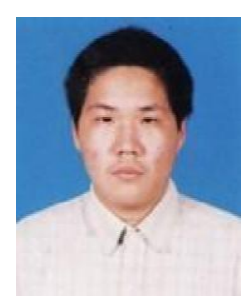

Agustinus Deddy Arief Wibowo received his bachelor degree in computer engineering from Multimedia University, Malaysia, in 2012. Currently he is pursuing master of engineering science in Multimedia University, Malaysia. His research interest includes pattern recognition, texture analysis and machine vision.

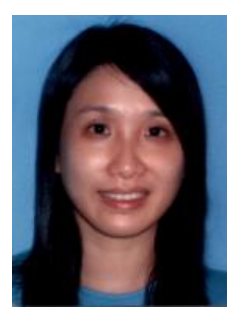

Sin Liang Lim received her bachelor of engineering, master in engineering, and $\mathrm{PhD}$ degrees from Multimedia University, RMIT (Royal Melbourne Institute of Technology) University, Australia, and Multimedia University, Malaysia in the years of 2001, 2003, and 2012 respectively. She joined Multimedia University as a lecturer in 2003 , and she is currently a senior lecturer in Faculty of Engineering, Cyberjaya campus in Multimedia University, Malaysia. With her $\mathrm{PhD}$ thesis entitled "Derivation of Novel Quantitative Characteristics from Geophysical Fields via Mathematical Morphology" Her research interests include terrestrial pattern retrieval using mathematical morphology, convexity measure and geodesic spectrum in digital topographic basins, and cloud field segmentation via multiscale convexity analysis.

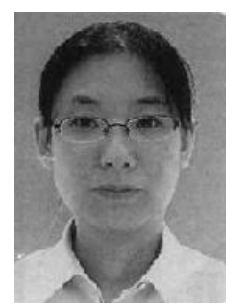

Wooi Nee Tan received a Comp.Sc.\&Ed.(Hons), M.Sc in mathematics and a Ph.D. degree in mathematics from the Technology University of Malaysia. Her research interests include mathematical modeling of dynamical systems and image processing. She is currently attached with Faculty of Engineering in Multimedia University as a lecturer. 